\title{
A Necessary and Sufficient Condition for Robust Asymptotic Stabilizability of Continuous-Time Uncertain Switched Linear Systems
}

\author{
Hai Lin and Panos J. Antsaklis
}

\begin{abstract}
The main contribution of this paper is a necessary and sufficient condition derived for the existence of asymptotically stabilizing switching laws for a class of switched linear systems with time-variant parametric uncertainties. This result improves upon the sufficient only conditions found in the literature. The method is based on polyhedral Lyapunovlike functions, which represent generalizations of polytopic Lyapunov functions in the classical sense.
\end{abstract}

\section{INTRODUCTION}

The stability issues of switched systems have been of increasing interest in the recent decade, see for example [5], [2] and the references cited therein. One of the most elusive problems in the switched systems literature has been the switching stabilizability problem, that is under what condition it is possible to stabilize a switched system by properly designing switching control laws.

In the switching stabilization literature, most of the work has focused on quadratic stabilization. For example, it was shown in [12] that the existence of a stable convex combination of two LTI subsystem matrices implies the existence of a state-dependent switching rule that stabilizes the switched system along with a quadratic Lyapunov function. A generalization to more than two LTI subsystems was introduced in [8] by using a "min-projection strategy". In [3], it was shown that the stable convex combination condition is also necessary for the quadratic stabilizability of two mode switched LTI system. However, it is only sufficient for switched LTI systems with more than two modes. A necessary and sufficient condition for quadratic stabilizability of switched controller systems was derived in [14]. There are extensions of [12] to output-dependent switching and discrete-time case [5], [16]. For robust stabilization, a quadratic stabilizing switching law was derived for polytopic uncertain switched linear systems based on LMI techniques in [16]. All of these methods guarantee stability by using a common quadratic Lyapunov function, which is conservative in the sense that there are switched systems that can be asymptotically (or exponentially) stabilized without using a common quadratic Lyapunov function. Therefore, some recent research efforts focused on using multiple Lyapunov functions. The first attempt of stabilizing switching law design based on multiple Lyapunov

The partial support of the National Science Foundation (NSF ECS9912458 \& CCR01-13131) is gratefully acknowledged. The first author appreciates the support from Center of Applied Mathematics Fellowship (2003-04), University of Notre Dame.

H. Lin and Panos J. Antsaklis are both with the Department of Electrical Engineering, University of Notre Dame, Notre Dame, IN 46556, USA. Email: $\{$ hlin1, antsaklis.1\}@nd.edu functions was proposed in [11], where piecewise quadratic Lyapunov functions was employed for two mode switched LTI systems. An LMI based method was proposed in [6] for the stabilizing state-feedback control design of discretetime piecewise affine systems. Exponential stabilization for switched LTI systems was considered in [9] also based on piecewise quadratic Lyapunov functions, and the synthesis problem was formulated as a bilinear matrix inequality (BMI) problem. In [4], a probabilistic algorithm was proposed for the synthesis of an asymptotically stabilizing switching law for switched LTI systems along with a piecewise quadratic Lyapunov function. Notice that these stabilizability conditions, which may be expressed as the feasibility of certain LMIs or BMIs, in the existing literature are basically sufficient only, except for certain cases of quadratic stabilization. Although necessary and sufficient conditions for asymptotic stabilizability of second-order switched LTI systems were derived in [15] by detailed vector field analysis, it was not apparent how to extend the method to either higher dimensions or to the parametric uncertainty case.

This paper focuses on this switching stabilizability problem, and introduces a necessary and sufficient condition for asymptotic stabilizability of switched linear systems with time-variant parametric uncertainties. The rest of the paper is organized as follows. In Section II, mathematical models for the uncertain switched linear system are described, and the robust switching stabilizability problem is formulated. Section III generalizes the classical polytopic Lyapunov functions to polyhedral Lyapunov-like functions so as to deal with the unstable subsystems. In Section IV, a necessary and sufficient condition for the asymptotic switching stabilizability is derived, which formulates the main result of the paper. In addition, the sufficiency proof is given in a constructive way, and provides a systematic method for stabilizing switching law synthesis. Finally, concluding remarks are presented and future work is proposed.

Notation: The letters $\mathcal{E}, \mathcal{P}, \mathcal{S} \cdots$ denote sets, $\partial \mathcal{P}$ the boundary of set $\mathcal{P}$, and $\operatorname{int}\{\mathcal{P}\}$ its interior. For any real $\lambda \geq 0$, the set $\lambda \mathcal{S}$ is defined as $\{x=\lambda y, y \in \mathcal{S}\}$. The term $\mathrm{C}$-set stands for a convex and compact set containing the origin in its interior.

\section{Problem Formulation}

We consider a collection of continuous-time linear systems described by the differential equations with parametric uncertainties

$$
\dot{x}(t)=A_{q}(w) x(t), \quad t \in \mathbb{R}^{+}, \quad q \in Q=\{1, \cdots, N\}
$$


where $\mathbb{R}^{+}$denotes non-negative real numbers. In the above uncertain continuous-time state equations, the state variable $x(t) \in \mathbb{R}^{n}$. Note that the origin $x_{e}=0$ is an equilibrium (maybe unstable) for the systems described in (1). The finite set $Q$ stands for the collection of discrete modes. In particular, for all $q \in Q, A_{q}(w): \mathcal{W} \rightarrow \mathbb{R}^{n \times n}$, and the entries of $A_{q}(w)$ are assumed to be continuous functions of $w \in \mathcal{W}$, where $\mathcal{W} \subset \mathbb{R}^{v}$ is a given compact set.

Combining the family of continuous-time uncertain linear systems (1) with a class of piecewise constant functions, $\sigma$ : $\mathbb{R}^{+} \rightarrow Q$, which serve as the switching signals between the collection of continuous-time systems (1). The continuoustime switched linear system can be described by

$$
\dot{x}(t)=A_{\sigma(t)}(w) x(t), \quad t \in \mathbb{R}^{+}
$$

where the switching signal is generated by

$$
\sigma(t)=\delta\left(\sigma\left(t^{-}\right), x(t)\right)
$$

with $\delta: Q \times \mathbb{R}^{n} \rightarrow Q$ and $t^{-}=\lim _{\tau \rightarrow 0, \tau \geq 0}(t-\tau)$. The discrete mode is determined by the current continuous state $x(t)$ and the previous mode $\sigma\left(t^{-}\right)$. It is assumed that there are finite switchings within any finite time interval.

For this uncertain continuous-time switched system (2)(3), we are interested in the following problem.

Problem: Given the continuous-time switched system (2), derive necessary and sufficient conditions, under which there exist switching control laws (3) that make the closedloop switched system globally asymptotically stable.

It is assumed that each subsystem (1) is unstable and satisfies the following assumption.

Assumption: There exists a full row rank matrix $L_{q} \in$ $\mathbb{R}^{m_{q} \times n}$, where $m_{q}<n$, such that the auxiliary system for the $q$-th subsystem (1)

$$
\dot{\xi}(t)=L_{q} A_{q}(w) R_{q} \xi(t), \quad t \in \mathbb{R}^{+}
$$

is asymptotically stable. Here $R_{q} \in \mathbb{R}^{n \times m_{q}}$ is a right inverse of $L_{q}$.

The above auxiliary system is derived through the generalized similarity transformation $R_{q} \xi=x$. Notice that even when all parts of the states of the original system (1) are unstable, there still may exist $L$ to satisfy the assumption.

Example 1: Consider a continuous-time linear system,

$$
\dot{x}(t)=\left[\begin{array}{cc}
0.5 & w \\
0 & 1
\end{array}\right] x(t)
$$

where the uncertain parameter $1 \leq w \leq 2$. The above continuous-time system is obviously unstable. However, we may select $L=\left[\begin{array}{ll}1 & 0\end{array}\right]$ and $R=\left[\begin{array}{c}1 \\ -1\end{array}\right]$ to obtain $L A(w) R=\left[\begin{array}{ll}1 & 0\end{array}\right]\left[\begin{array}{cc}0.5 & w \\ 0 & 1\end{array}\right]\left[\begin{array}{c}1 \\ -1\end{array}\right]=0.5-w<0$, for all $w \in[1,2]$. Therefore, the auxiliary system

$$
\dot{\xi}(t)=(0.5-w) \xi(t)
$$

is asymptotically stable. In fact, there may exist more than one pair of matrices $L_{q}$ and $R_{q}$ that satisfy the above assumption. For example, one may pick another $R=$ $\left[\begin{array}{c}1 \\ -a\end{array}\right]$ with $a>0.5$, to obtain a stable auxiliary system

$$
\dot{\xi}(t)=(0.5-a w) \xi(t) .
$$

Based on the existence of the stable auxiliary system, we may conclude that all the states $x$ contained in the range space of $R$ asymptotically converge to the null space of $L=\left[\begin{array}{ll}1 & 0\end{array}\right]$, i.e., the $y$-axis. The partial convergence property of the original system is captured by a polyhedral Lyapunov-like function that is developed in the next section.

It can be shown that for the LTI case ${ }^{1}, \dot{x}(t)=A x(t)$, there always exist $L$ and $R$ satisfying the above assumption, except for the case when all the eigenvalues of $A$ are the same positive real number $\lambda>0$ and the geometric multiplicity of the eigenvalue $\lambda$ equals $n$. The proof of this claim explores the structure of the Jordan canonical form of $A$ and uses straight-forward computations. Details are omitted here.

For the case that there does not exist $L$ to satisfy the above assumption for a particular subsystem, we simply set $L$ as the null row vector, which implies that the corresponding subsystem makes no contribution to the stabilization of the switched system. To justify this, note that in this case the matrix $A$ is similar to the matrix $\lambda I$ for some positive real number $\lambda>0$. Here $I$ stands for the identity matrix. If we look at the phase plane of the LTI system, $\dot{x}(t)=\lambda I x(t)$, all the field vectors point to infinity along the radial directions. Intuitively speaking, the dynamics are explosive and do nothing but drag all the states to infinity. Therefore, we set $L$ to be a null vector so to contribute nothing to the rank condition in Theorem 1 below.

\section{PolyhedRAL LyAPUNOV-LIKE FunCtions}

It is known that the robust asymptotic stability of timevarying linear systems implies the existence of a polytopic Lyapunov function [7]. Therefore, the asymptotic stability of the auxiliary system (4) in $\mathbb{R}^{m_{q}}$ implies the existence of a polytopic Lyapunov function

$$
\Phi_{q}(\xi)=\max _{1 \leq i \leq s_{q}}\left\{f_{i} \xi\right\}
$$

which can be constructed by either algebraic or numerical methods. Let $F_{q} \in \mathbb{R}^{s_{q} \times m_{q}}\left(s_{q} \geq m_{q}\right)$ be the matrix with $f_{i} \in \mathbb{R}^{1 \times m_{q}}$ as its $i$-th row vector. The Lyapunov level set

$\mathcal{P}_{q}=\left\{\xi \in \mathbb{R}^{m_{q}}: \Phi_{q}(\xi) \leq 1\right\}=\left\{\xi \in \mathbb{R}^{m_{q}}: F_{q} \xi \leq \overline{1}\right\}$

is an invariant set [1], where $\overline{1}$ stands for a column vector in $\mathbb{R}^{m_{q}}$ with all elements being 1 and $\leq$ is component-wise.

The next step is to shape the polytopic Lyapunov function $\Phi_{q}(\xi)$ for the auxiliary system (4) into a polyhedral Lyapunov-like function for the original system (1).

\footnotetext{
${ }^{1}$ This corresponds to the uncertain parameter set $\mathcal{W}$ being a singleton.
} 
For this, we need to introduce the Euler Approximate System (EAS) for the auxiliary system (4):

$$
\xi[k+1]=L_{q}\left[I+\tau A_{q}(w)\right] R_{q} \xi[k], \quad k \in \mathbb{Z}^{+} .
$$

The connection between the continuous-time system (4) and its corresponding discrete-time EAS (6) is based on the concept of a contractive set.

Definition 1: Given a positive scalar $\lambda, 0<\lambda<1$, a set $\mathcal{P}$ is said $\lambda$-contractive with respect to the discrete-time EAS (6), if, for any $\xi \in \mathcal{P}$, all the possible next step states through the transition (6) are contained in the set $\lambda \mathcal{P}$, that is

$$
L_{q}\left[I+\tau A_{q}(w)\right] R_{q} \xi \in \lambda \mathcal{P},
$$

holds for any $w \in \mathcal{W}$.

It is shown in [1], that for an asymptotically stable system (4), there always exist a positive scalar $\bar{\tau}>0$ and a positive constant $\lambda<1$ such that, for all $0<\tau \leq \bar{\tau}$, the level set $\mathcal{P}_{q}=\left\{\xi: F_{q} \xi \leq \overline{1}\right\}$ in (5) is $\lambda$-contractive for the corresponding discrete-time EAS (6).

Therefore, we obtain that

$$
F_{q} L_{q}\left[I+\tau A_{q}(w)\right] R_{q} \xi \leq \lambda \overline{1}
$$

holds for all $\xi \in \mathcal{P}_{q}=\left\{\xi: F_{q} \xi \leq \overline{1}\right\}$, for all $w \in \mathcal{W}$ and for $\tau$ small enough.

For all the states $x$ contained in the range space of $R_{q}$, one may represent $x=R_{q} \xi$. Note that the range space of $R_{q}$, denoted as image $\left(R_{q}\right)$, is a linear subspace of $\mathbb{R}^{n}$. Hence, for all $x \in\left\{x: F_{q} L_{q} x \leq \overline{1}\right\} \bigcap \operatorname{image}\left(R_{q}\right)$,

$$
F_{q} L_{q}\left[I+\tau A_{q}(w)\right] x \leq \lambda \overline{1}
$$

holds for all $w \in \mathcal{W}$ and for all $0<\tau \leq \bar{\tau}$. As it has been pointed out in Example 1, the matrix $R_{q}$ that satisfies the assumption (4) and admits $\mathcal{P}_{q}$ as a $\lambda$-contractive set, is not unique for a given $L_{q}$. It is straight forward to verify that all the states in the union of the range space of these $R_{q}$, denoted as

$$
\mathcal{M}_{q}=\bigcup_{R_{q}} \operatorname{image}\left(R_{q}\right),
$$

also have the convergence property, that is for all $x \in\{x$ : $\left.F_{q} L_{q} x \leq \overline{1}\right\} \bigcap \mathcal{M}_{q}$

$$
F_{q} L_{q}\left[I+\tau A_{q}(w)\right] x \leq \lambda \overline{1}
$$

for all $w \in \mathcal{W}$ and for all $0<\tau \leq \bar{\tau}$. This case is referred to as the set

$$
\mathcal{S}_{q}=\left\{x: F_{q} L_{q} x \leq \overline{1}\right\},
$$

which may be an unbounded polyhedral set, is a partial $\lambda$-contractive set for the discrete-time system

$$
x[k+1]=\left[I+\tau A_{q}(w)\right] x[k]
$$

along $\mathcal{M}_{q}$. If the set $\mathcal{M}_{q}$, which contains a union of linear subspaces of $\mathbb{R}^{n}$, is the whole state space $\mathbb{R}^{n}$, then we obtain the usual contractiveness for the set $\mathcal{S}_{q}$ with respect to (9).
Notice that the above discrete-time system (9) is the EAS of the original subsystem (1). In the following, we will show that the existence of such partial contractive set $\mathcal{S}_{q}$ for the EAS (9) implies a polyhedral Lyapunov-like function, which is defined below, for the subsystem (1).

Denote $F_{q} L_{q} \in \mathbb{R}^{s_{q} \times n}$ as $H_{q}$, and $h_{i}$ as the $i$-th row vector of $H_{q}$. Then the polyhedral Lyapunov-like function candidate from the polyhedron $\mathcal{S}_{q}$ can be defined as

$$
\Psi_{q}(x)=\max _{1 \leq i \leq s_{q}}\left\{h_{i} x, 0\right\} .
$$

It is straightforward to verify that $\Psi_{q}(x) \geq 0$ for all $x \in$ $\mathbb{R}^{n}$, and that $\Psi_{q}(x)$ is convex, continuous and piecewise linear for $x$. However, $\Psi_{q}(x)=0$ does not imply that $x$ is the origin. In fact, for all $x$ contained in the convex cone

$$
\mathcal{C}_{q}=\left\{x: H_{q} x \leq \overline{0}\right\},
$$

we have $\Psi_{q}(x)=0$. This is one of the main differences from the classical Lyapunov function, so we call $\Psi_{q}(x)$ a Lyapunov-like function.

Next, we will show that the Dini derivative of $\Psi_{q}$ along the trajectory of the continuous-time system (1) is negative for all $x$ contained in $\mathcal{M}_{q}$ and outside the cone $\mathcal{C}_{q}$, where the Dini derivative $\mathcal{D}^{+} \Psi_{q}(x(t))$ is defined as

$$
\mathcal{D}^{+} \Psi_{q}(x(t))=\lim \sup _{\tau \rightarrow 0, \tau \geq 0} \frac{\Psi_{q}(x(t+\tau))-\Psi_{q}(x(t))}{\tau} .
$$

It was shown in [1] that the Dini derivative of $\Psi_{q}$ at the time instant $t$, for $x(t)=x$, and $w(t)=w$, can be calculated as

$$
\mathcal{D}^{+} \Psi_{q}(x(t))=\lim \sup _{\tau \rightarrow 0, \tau \geq 0} \frac{\Psi_{q}\left(x+\tau A_{q}(w) x\right)-\Psi_{q}(x)}{\tau} .
$$

The following property of the partial contractive sets for EAS (9) is essential to prove that the Dini derivative of $\Psi_{q}$ is negative along the trajectory of (1).

Lemma 1: If $\mathcal{S}$ is a partial $\lambda$-contractive set for the EAS (9) along $\mathcal{M}_{q}$, then $\mu \mathcal{S}$ is so for all $\mu>0$.

Proof : First, for all $x \in \mu \mathcal{S} \cap \mathcal{M}_{q}$ and any positive scalar $\mu>0, \mu^{-1} x \in \mathcal{S} \cap \mathcal{M}_{q}$, because of the fact that $\mathcal{M}_{q}$ is a union of linear subspaces. Secondly, for such state $x \in$ $\mu \mathcal{S} \cap \mathcal{M}_{q}$, the following inequality

$$
F_{q} L_{q}\left[I+\tau A_{q}(w)\right] x=\mu F_{q} L_{q}\left[I+\tau A_{q}(w)\right] \mu^{-1} x \leq \mu \lambda \overline{1}
$$

holds for all $w \in \mathcal{W}$ and for all $0<\tau \leq \bar{\tau}$. This implies that the set $\mu \mathcal{S}$ is a partial $\lambda$-contractive set for the EAS (9) along $\mathcal{M}_{q}$.

The next lemma shows that the partial contractiveness of the polyhedral set $\mathcal{S}_{q}$ for the EAS (9) implies the negativeness of the Dini derivative of $\Psi_{q}$ for (1). The proof is omitted here for space limit.

Lemma 2: If there exist scalars $0<\lambda<1$ and $\bar{\tau}>0$, such that the polyhedral set $\mathcal{S}_{q}=\left\{x: H_{q} x \leq \overline{1}\right\}$ is a partial $\lambda$-contractive set for the EAS (9) along $\mathcal{M}_{q}$ with all $0<\tau<\bar{\tau}$, then the Dini derivative $\mathcal{D}^{+} \Psi_{q}(x(t))$ for all $x(t)$ contained in $\mathcal{M}_{q}$ and outside the cone $\mathcal{C}_{q}$ is negative along the trajectory of the continuous-time system (1). 
In summary, we started from Assumption (4) and proved the existence of a polyhedral Lyapunov-like function $\Psi_{q}(x(t))$ for the subsystems (1). The determination of such polyhedral Lyapunov-like function can be reduced to the determination of a polytopic Lyapunov function of the auxiliary system (4) if the matrix $L_{q}$ is given. It was shown in [1] that a polytopic Lyapunov function may be derived by numerical algorithms involving polyhedral sets. To calculate the matrix $L_{q}$ that satisfies the assumption, a systematic method can be developed for the LTI case by exploring the Jordan canonical form. In the next section, we will derive a necessary and sufficient condition for the asymptotic stabilizability of the switched linear system (2)(3). The techniques are based on the polyhedral Lyapunovlike functions developed here.

\section{Main Results}

First, we introduce some notations corresponding to the intersection of the polyhedral sets $\mathcal{S}_{q}$ in (8) for $q \in Q$. Assume that $\mathcal{S}=\bigcap_{q \in Q} \mathcal{S}_{q}$ is a bounded polyhedral set with the origin in its interior, i.e., a polyhedral C-set. Let $\operatorname{vert}(\mathcal{S})=\left\{v_{1}, v_{2}, \cdots, v_{e}\right\}$ stand for its finite vertices, while $\operatorname{face}(\mathcal{S})=\left\{F_{1}, F_{2}, \cdots, F_{M}\right\}$ denote its facets. The hyperplane that corresponds to the $k$-th facet $F_{k}$ is defined by

$$
H_{k}=\left\{x \in \mathbb{R}^{n}: f_{k} x=1\right\}
$$

where $f_{k} \in \mathbb{R}^{1 \times n}$ is the corresponding gradient vector of facet $F_{k}$. The set of vertices of $F_{k}$ can be found as $\operatorname{vert}\left(F_{k}\right)=\operatorname{vert}(\mathcal{P}) \cap F_{k}$. We denote the cone generated by the vertices of $F_{k}$ by

$$
\operatorname{cone}\left(F_{k}\right)=\left\{x: \sum_{i} \alpha_{i} v_{k_{i}}, \alpha_{i} \geq 0, v_{k_{i}} \in \operatorname{vert}\left(F_{k}\right)\right\}
$$

Note that if $\mathcal{S}$ is a polyhedral $\mathrm{C}$-set, then the union of the cones is the whole state space, i.e., $\bigcup_{k=1, \cdots, M} \operatorname{cone}\left(F_{k}\right)=$ $\mathbb{R}^{n}$.

For each facet of $\mathcal{S}, F_{k}$, there exists at least one mode $q$ such that the gradient vector of facet $F_{k}$, namely $f_{k}$, is one of the (non-redundant) row vectors of $H_{q}$. This is simply because of the fact that $\mathcal{S}$ is the intersection of $\mathcal{S}_{q}$ for $q \in Q$. Collect all such modes $q$ and call them active modes for $\operatorname{cone}\left(F_{k}\right)$, which are denoted as $\operatorname{Act}\left\{\operatorname{cone}\left(F_{k}\right)\right\}$.

It can be shown that these active modes in $\operatorname{cone}\left(F_{k}\right)$ have the following properties. First, for two different modes $q_{1}$, $q_{2} \in \operatorname{Act}\left\{\operatorname{cone}\left(F_{k}\right)\right\}$, the equality $\Psi_{q_{1}}(x)=\Psi_{q_{2}}(x)$ holds for all $x \in \operatorname{cone}\left(F_{k}\right)$. Secondly, for any $q \in \operatorname{Act}\left\{\operatorname{cone}\left(F_{k}\right)\right\}$ and any another mode $q^{\prime} \in Q, \Psi_{q}(x) \geq \Psi_{q^{\prime}}(x)$ holds for all $x \in \operatorname{cone}\left(F_{k}\right)$. The results are not surprising, if one considers the geometric interpretation of the Lyapunov-like function which is basically a distance measure from a point to the boundaries of a polyhedral set.

\section{A. Main Theorem}

A necessary and sufficient condition for the robust asymptotic stabilizability of the uncertain switched linear systems (2)-(3) can be stated as the following theorem.
Theorem 1: The switched linear system (2)-(3) with time-variant uncertainties can be globally asymptotically stabilized by a switching law, if and only if there exist matrices $L_{q}$, which satisfy the assumption (4) for each subsystem respectively, such that the matrix

$$
\left[\begin{array}{c}
L_{1} \\
L_{2} \\
\vdots \\
L_{N}
\end{array}\right] \in \mathbb{R}^{\sum_{q} m_{q} \times n}
$$

has $n$ linear independent row vectors, and

$$
\bigcup_{q \in Q} \Omega^{q}=\mathbb{R}^{n}
$$

where $\Omega^{q}$ are the union of the cones $\operatorname{cone}\left(F_{k}\right)$ (12) that $q \in \operatorname{Act}\left\{\operatorname{cone}\left(F_{k}\right)\right\}$ and $\operatorname{cone}\left(F_{k}\right) \subseteq \mathcal{M}_{q}(8)$.

\section{B. Sufficiency of Theorem 1}

This subsection is devoted to the sufficiency proof of Theorem 1, which is given in a constructive way. In the following, a stabilizing switching law is constructed and a global Lyapunov function is composed for the switched system. The techniques are based on the polyhedral Lyapunovlike function $\Psi_{q}(x(t))$ for each (unstable) subsystem.

First, we give a necessary and sufficient condition for a 0 -symmetric polyhedrons, i.e., if $x \in \mathcal{P}$ then $-x \in \mathcal{P}$, to be bounded. Note that a bounded polyhedron is usually called a polytope.

Lemma 3: A non-empty 0-symmetric polyhedral set

$$
\mathcal{P}=\left\{x \in \mathbb{R}^{n}:|H x| \leq g\right\}
$$

is bounded, if and only if the matrix $H \in \mathbb{R}^{s \times n}(s \geq n)$ has $n$ linear independent row vectors, or equivalently the rank of $H$ equals $n$.

In particular, for the intersection of $\mathcal{S}_{q}$, we have the following corollary.

Corollary 1: If all the polyhedral sets $\mathcal{P}_{q}$ in $\mathbb{R}^{m_{q}}$ are 0-symmetric, then so are the $\mathcal{S}_{q}$ in $\mathbb{R}^{n}$, for all $q \in$ $Q=\{1,2, \cdots, N\}$. In addition, the intersection of all the polyhedral sets $\mathcal{S}_{q}$ is bounded, if and only if the matrix (13) has $n$ linear independent row vectors, or equivalently it has rank $n$.

From Corollary 1, we obtain that the rank condition (13) implies that the intersection of $\mathcal{S}_{q}$, that is the set $\mathcal{S}$, is a polyhedral $\mathrm{C}$-set. Therefore, we may partition the state space $\mathbb{R}^{n}$ into finite cones which are induced from the facets of $\mathcal{S}$, and relabel these cones as corresponding $\Omega^{q}$. Because of the condition (14), a conic partition of the state space $\mathbb{R}^{n}$ is given by $\Omega^{q}, q \in Q$. We define the switching law as:

$$
x \in \Omega^{q} \Rightarrow \delta(\cdot, x)=q
$$

It can be shown that the switching law defined above can guarantee the uniformly ultimate boundedness (UUB) for the uncertain switched system (2)-(3) into $\mathcal{S}=\bigcap_{q \in Q} \mathcal{S}_{q}$. Basically, the term UUB means all the trajectories would 
converge to $\mathcal{S}$ and stay inside $\mathcal{S}$, which can be guaranteed by the existence of a Lyapunov function outside $\mathcal{S}$ [1].

Proposition 1: Consider the class of switching laws defined by $\delta(\cdot, x)=q$ if $x$ is contained in $\Omega^{q}$. Then, the uncertain continuous-time switched system (2)-(3) is UUB in the polyhedral C-set $\bigcap_{q \in Q} \mathcal{S}_{q}$.

Proof: Define the function $V(x)=\max _{q \in Q} \Psi_{q}(x)$. For all $x(t) \notin \bigcap_{q \in Q} \mathcal{S}_{q}, V(x(t))=\max _{q \in Q} \Psi_{q}(x)>1$. Assume that $x(t) \in \Omega^{q}$ and current mode $\sigma(t)=q$. If no switching occurs at $t$, then there exists $\bar{\tau}>0$ such that $\forall 0<\tau \leq \bar{\tau}$, $x(t+\tau) \in \Omega^{q}$ and $x(t+\tau) \notin \operatorname{int}\left(\mathcal{S}_{q}\right)$. Then $V(x(t))=$ $\max _{q \in Q} \Psi_{q}(x(t))=\Psi_{q}(x(t))$ and $V(x(t+\tau))=\Psi_{q}(x(t+$ $\tau))$. Then, according to Lemma 2

$$
\mathcal{D}^{+} V(x(t))=\mathcal{D}^{+} \Psi_{q}(x(t))<0
$$

Else, if switching occurs at time $t$, then there exists $\bar{\tau}>$ Osuch that $\forall 0<\tau \leq \bar{\tau}, x(t+\tau) \in \Omega_{q^{\prime}}$ and $x(t+$ $\tau) \notin \operatorname{int}\left(\mathcal{S}_{q^{\prime}}\right)$. Then $V(x(t))=\max _{q \in Q} \Psi_{q}(x(t))=$ $\Psi_{q}(x(t))=\Psi_{q^{\prime}}(x(t))$ and $V(x(t+\tau))=\Psi_{q^{\prime}}(x(t+\tau))$. Therefore, according to Lemma 2

$$
\mathcal{D}^{+} V(x(t))=\lim \sup _{\tau \rightarrow 0^{+}} \frac{\Psi_{q^{\prime}}(x(t+\tau))-\Psi_{q^{\prime}}(x(t))}{\tau}<0 .
$$

Therefore, the uncertain switched system (2)-(3) is UUB with respect to the region $\bigcap_{q \in Q} \mathcal{S}_{q}$.

Because of Lemma 1 and the above UUB result, the switching control law can drive all the state trajectories into $\mu \mathcal{S}$ for all $\mu>0$ within a finite time interval. Select any decreasing sequence of $\mu_{k}$ with $\lim _{k \rightarrow \infty} \mu_{k}=0$; then all the trajectories will finally be driven to the origin. This implies globally asymptotic stability for the switched linear system (2)-(3).

The last point for the sufficiency proof is to show that it is always possible to pick 0 -symmetric polyhedral set $\mathcal{S}_{q}$. This is simply because that the asymptotic stability of the $q$ th auxiliary system (4) implies the existence of a polytopic Lyapunov function $\Psi_{q}$ in the infinite norm form [7], for example

$$
\Psi_{q}(\xi)=\left\|F_{q} \xi\right\|_{\infty}
$$

By the arguments in the previous section, the polytopic Lyapunov function $\Psi_{q}$ implies a contractive polytope for the EAS (6), which can be represented as

$$
\mathcal{P}_{q}=\left\{\xi \in \mathbb{R}^{m_{q}}:\left|F_{q} \xi\right| \leq \overline{1}\right\} .
$$

So the generated contractive polyhedral for the original system (1) in $\mathbb{R}^{n}$ can be represented as

$$
\mathcal{S}_{q}=\left\{x \in \mathbb{R}^{n}:\left|F_{q} L_{q} x\right| \leq \overline{1}\right\},
$$

which is 0 -symmetric. With the satisfaction of the rank condition (13), we know that $\mathcal{S}=\bigcap_{q} \mathcal{S}_{q}$ is a C-set by Corollary 1. Therefore, a conic partition based stabilizing switching law can be constructed, and a global Lyapunov function $V(x)$ can be composed as above.

This completes the sufficiency proof of Theorem 1 .

\section{Necessity of Theorem 1}

To show the necessity of Theorem 1, we need the following lemma.

Lemma 4: A switched system can be globally asymptotically stabilized by a switching law if and only if it can be stabilized by a conic partition switching law.

Proof : Because of the fact that a conic partition switching law is a specific class of switching law, the necessity is obvious.

To prove sufficiency, it is assumed that the switched system can be globally asymptotically stabilized by a properly designed switching law. Therefore, there exists a switching signal $\sigma(t)$ such that the closed-loop switched system

$$
\dot{x}(t)=A_{\sigma(t)}(w) x(t)
$$

is globally asymptotically stable. Then, a polytopic Lyapunov function $\Psi(x)$ for the closed-loop switched linear system exists [7], and its level set

$$
\mathcal{P}=\left\{x \in R^{n}: \Psi(x) \leq 1\right\}
$$

is a C-set [1].

For any $x \in \partial \mathcal{P}$, according to the above asymptotic stability assumption, there exists at least one mode $q$ such that the Dini derivative of $\Psi(x)$ is negative along the dynamics of mode $q$. Similar to the arguments in [1], there exists a positive constant $\bar{\tau}>0$ and a scalar $0<\lambda<1$, such that

$$
\left[I+\tau A_{q}(w)\right] x \subset \lambda \mathcal{P}
$$

holds for all $0<\tau \leq \bar{\tau}$. In addition, for any positive scalar $\mu>0$,

$$
\left[I+\tau A_{q}(w)\right] \mu x \subset \lambda \mu \mathcal{P},
$$

holds for all $0<\tau \leq \bar{\tau}$. This implies that the Dini derivative of $\Psi(\mu x)$ is negative along the dynamics of mode $q$ [1].

Because of the continuity, there exists a small neighborhood of $x, B_{r}(x)$, such that for all $y \in \partial \mathcal{P} \cap B_{r}(x)$,

$$
\left[I+\tau A_{q}(w)\right] y \subset \lambda \mathcal{P},
$$

holds for all $0<\tau \leq \bar{\tau}$. Note that

$$
\partial \mathcal{P} \subseteq \bigcup_{x \in \partial \mathcal{P}} \partial \mathcal{P} \cap B_{r}(x)
$$

and the fact that $\partial \mathcal{P}$ is closed and bounded in $\mathcal{R}^{n}$, namely compact. Therefore, there exists a finite cover for $\partial \mathcal{P}$, which induces a finite partition of the faces of $\mathcal{P}$. With each partition of $\partial \mathcal{P}$, a conic cone can be generated and the union of these cones is the whole state space. Within each cone, following the previous arguments, one mode $q$ can be selected to make the Dini derivative of $\Psi(x)$ negative along the dynamics of mode $q$. This generates a conic switching law which globally asymptotically stabilizes the switched system.

Because of the above lemma, the existence of an asymptotically stabilizing switching law for the switched system (2)-(3) implies the existence of a conic partition based 
switching law which globally asymptotically stabilizes the closed-loop switched system. In the following, we will show that the existence of such conic partition based stabilizing switching law implies (13) and (14).

First, we show that (14) is necessary. Assume that this is not the case. Hence, there exists a point $\hat{x}$ such that the Dini derivative of $\Psi(\hat{x})$ is negative along the mode $q$ but the point $\hat{x}$ is not contained in the union of range spaces $\mathcal{M}_{q}$. Similarly to the arguments in Lemma 4 , it can be shown that the Dini derivative of $\Psi(x)$ is negative along the mode $q$ for all the points $x=\mu \hat{x}$ with $\mu>0$. If the level set of $\Psi(x), \mathcal{P}$, is 0 -symmetric, then the negativeness of the Dini derivative of $\Psi(x)$ along mode $q$ holds for all $\mu \hat{x}$ with $\mu \neq 0$. Note that one may always pick a 0 -symmetric polytope $\mathcal{P}$ without loss of generality [7]. All the points $\mu \hat{x}$ form a linear subspace in $\mathbb{R}^{n}$, and the scalar $\mu$ equals zero corresponds to the origin. This linear subspace can be represented as a range space of a vector $R_{q}$. Notice that $\Psi(x)$ is piecewise linear and can be represented in the following form [7]

$$
\Psi(x)=\max _{1 \leq i \leq s}\left\{\left|f_{i} x\right|\right\} .
$$

Denote $i_{0}=\arg \max _{1 \leq i \leq s}\left\{\left|f_{i} \mu \hat{x}\right|\right\}$ and select the row vector $f_{i_{0}}$ as $L_{q}$. Then, the negativeness of the Dini derivative of $\Psi(x)$, in fact $\left|f_{i} \mu \hat{x}\right|$, along mode $q$ for all $\mu \hat{x}$, implies that the auxiliary system

$$
\dot{\xi}(t)=L_{q} A_{q}(w) R_{q} \xi(t)
$$

is asymptotically stable. The condition that $L_{q} R_{q}=1$ can be easily satisfied by a scaling factor since $L_{q} R_{q} \neq 0$. This implies that the point $\hat{x}$ is contained in the union of range space $\mathcal{M}_{q}$, which leads to a contradiction.

Next, we show that the rank condition (13) is necessary for the construction of a conic partition switching law. Let us denote the conic partition derived in the proof of Lemma 4 as $\Omega^{q}$, then

$$
\bigcup_{q \in Q} \Omega^{q}=\mathbb{R}^{n} .
$$

As proved in Lemma 4, within the cone $\Omega^{q}$, the Dini derivative of $\Psi(x)$ is negative along the dynamics of the mode $q$. This means that the cone

$$
\mathcal{C}=\bigcap_{q} \mathcal{C}_{q}
$$

contains only the null vector, which implies that the intersection of $\mathcal{S}_{q}(q \in Q)$ is bounded. By Corollary 1, the rank condition (13) is obtained.

This completes the necessity proof.

\section{CONCLUDING REMARKS}

In this paper, continuous-time switched linear systems affected by parameter variations were considered. The robust stabilizability problem for such uncertain switched linear systems was investigated. A necessary and sufficient condition for the existence of a switching control law to assure the asymptotic stability of the closed-loop switched systems was derived. The sufficiency proof also led to a constructive method for the stabilizing switching law synthesis, which is characterized by a conic partition of the state space.

The generalized similarity transformation (4), especially in the parametric uncertainty case, needs to be better understood and is currently under study. Two problems are of particular interest. One is the existence of a stable generalized system similar to (1) with uncertainties. The second problem is how to develop an efficient method to determine or to parameterize the similarity transformation matrix $L_{q}$ for a given subsystem (1).

\section{ACKNOWLEDGEMENTS}

The authors would like to thank Dr. Ray DeCarlo, Dr. Magnus Egerstedt, Dr. João Pedro Hespanha, Dr. Daniel Liberzon, Mr. Qiang Ling, and Dr. Guisheng Zhai for their comments.

\section{REFERENCES}

[1] F. Blanchini, "Nonquadratic Lyapunov functions for robust control," Automatica, vol. 31, pp. 451-461, 1995.

[2] R. A. Decarlo, M. S. Branicky, S. Pettersson, and B. Lennartson, "Perspectives and results on the stability and stabilizability of hybrid systems," Proccedings of the IEEE, vol. 88, no. 7, pp. 1069-1082, 2000.

[3] E. Feron, "Quadratic stabilizability of switched systems via state and output feedback," technique report, CICS-P-468, MIT, 1996.

[4] H. Ishii, T. Basar, and R. Tempo, "Synthesis of switching rules for switched linear systems through randomized algorithms," in Proc. 42nd IEEE Conf. Decision Control, pp. 4788-4793, 2003.

[5] D. Liberzon and A. S. Morse, "Basic problems in stability and design of switched systems," IEEE Contr. Syst. Magazine, vol. 19, no. 15 , pp. 59-70, 1999.

[6] D. Mignone, G. Ferrari-Trecate, and M. Morari, "Stability and stabilization of piecewise affine and hybrid systems: An LMI approach," in Proc. 39th Conf. Decision Control, pp. 504-509, 2000.

[7] D. Liu and A. Molchanov, "Criteria for robust absolute stability of time-varying nonlinear continuous-time systems," Automatica, vol. 38, no. 4, pp. 627-637, 2002.

[8] S. Pettersson and B. Lennartson, "Stabilization of hybrid systems using a min-projection strategy," in Proc. 2001 American Contr. Conf., pp. 223-228, 2001.

[9] S. Pettersson, "Synthesis of switched linear systems," in Proc. 42nd IEEE Conf. Decision Control, pp. 5283-5288, 2003.

[10] M. A. Wicks, P. Peleties, and R. A. DeCarlo, "Construction of piecewise Lyapunov function for stabilizing switched systems," in Proc. 35th Conf. Decision Control, pp. 3492-3497, 1994.

[11] M. A. Wicks and R. A. DeCarlo, "Solution of coupled Lyapunov equations for the stabilization of multimodal linear systems," in Proc. 1997 American Contr. Conf., pp. 1709-1713, 1997.

[12] M. A. Wicks, P. Peleties, and R. A. DeCarlo, "Switched controller design for the quadratic stabilization of a pair of unstable linear systems," European J. Control, vol. 4, pp. 140-147, 1998.

[13] R. N. Shorten and K. S. Narendra, "On the stability and existence of common Lyapunov functions for stable linear switching systems," in Proc. 37th Conf. Decision Control, pp. 3723-3724, 1998.

[14] E. Skafidas, R. J. Evans, A. V. Savkin, and I. R. Petersen, "Stability results for switched controller systems," Automatica, vol. 35, no. 4, pp. 553-564, 1999.

[15] X. Xu and P. J. Antsaklis, "Stabilization of second-order LTI switched systems," Inter. J. Contr., vol. 73, no. 14, pp. 1261-1279, 2000.

[16] G. Zhai, H. Lin, and P. J. Antsaklis, "Quadratic stabilizability of switched linear systems with polytopic uncertainties," Inter. J. Contr., vol. 76, no. 7, pp. 747-753, 2003. 